\title{
Cabral, Césaire, Du Bois : Trois Voix Atlantiques pour Amplifier la Portée des Luttes d'Émancipation
}

\author{
Fabrice Schurmans \\ Université de Coimbra-Centre d'Études Sociales
}

Résumé: L'Atlantique noir est devenu un outil de référence pour les études postcoloniales. La notion fait sens lorsqu'il s'agit d'étudier les présences de l'hyperviolence et des luttes d'émancipation dans des œuvres littéraires et des parcours politiques. Nous reviendrons d'abord sur le lien entre l'Atlantique et la notion d'identité. Ce lieu où se donne à voir, à lire et à entendre une multitude de biens symboliques, tend en effet à favoriser des identités excentrées, moins marquées par un nous enraciné et exclusif. Cet article se penchera ensuite sur la façon dont Cabral, Césaire et Du Bois ont, à partir de cet espace symbolique, pensé les formes de l'émancipation. Envisagée à partir du Sud global, l'émancipation requière un espace analytique dépassant le cadre local. Nous montrerons que l'Atlantique s'impose non comme une ressource parmi d'autres, mais comme une nécessité face à la nature de l'objet étudié.

Mots-clés: Atlantique noir, Cabral, Césaire, Du Bois, émancipation

\begin{abstract}
The Black Atlantic has become a key reference in postcolonial studies. This concept is extremely useful to examine the presence of hyperviolence and struggles for emancipation in literary works and political paths. This article addresses the link between the Atlantic and the notion of identity. This metaphoric place, where a wide variety of symbolic goods circulates, tends indeed to favour dynamic processes of identification, less marked by a rooted and exclusive Us. Then, this article examines how Cabral, Césaire and Du Bois have, from that symbolic space, thought the forms of emancipation. When considered from the global South, emancipation requires an analytic space that goes beyond the local setting. This article argues that the Atlantic imposes itself not as a resource among others, but as a necessity given the nature of the object being studied.
\end{abstract}

Keywords: Black Atlantic, Cabral, Césaire, Du Bois, emancipation 
Il est connu que l'Atlantique noir constitue un outil heuristique puissant lorsqu'il s'agit d'analyser certains biens symboliques postcoloniaux. Comme l'indiquent les contributions à un numéro de Politique Africaine consacré à la "Blackness",1 la photographie, le rap, la littérature issus des mondes atlantiques sont porteurs d'une esthétique particulière que l'on peut interpréter à partir des notions d'hybridité, de métissage, de frontière(s), d'identité(s), d'itinérance. ${ }^{2}$ Cependant, parce qu'il s'agit d'un contexte marqué par diverses formes de violence et d'exploitation, ces mêmes biens symboliques sont également, à des degrés divers, porteurs d'une éthique propre, déterminée par les luttes d'émancipation et leurs conséquences. Envisagée à partir de la métaphore de l'Atlantique noir, l'émancipation renvoie à des formes de coercition semblables d'une rive à l'autre et autorise un rapprochement entre des auteurs écrivant à partir de contextes différents. Cet article examinera la façon dont trois intellectuels de premier plan ont approché la notion en question : Amilcar Cabral (O Papel da Cultura na Luta pela Independência, 1974), Aimé Césaire (Discours sur le Colonialisme, 1955) et W.E.B Du Bois (The Souls of Black Folk, 1903).

Revenir à Cabral, Césaire et Du Bois permet de saisir le lien existant entre les penseurs critiques issus des diasporas africaines et de comprendre l'actualité d'une philosophie concrète de l'émancipation. D’une certaine façon, leurs textes dialoguent, entretiennent une espèce de fil conducteur les reliant les uns aux autres, cela non seulement parce que Cabral et Césaire ont lu Du Bois, mais également parce que confrontés, indépendamment de leurs contextes sociaux respectifs, à une oppression violente et de longue durée, ils y ont apporté des réponses souvent similaires. Il s'agit aussi de reprendre ces textes au XXIe siècle afin de montrer leur pertinence au moment où la parole et le geste racistes redeviennent visibles, dans l'espace public autant que dans le discours social et politique. Sur ce point, on se souviendra que Balibar et Wallerstein avaient déjà pointé l'émergence et la sédimentation d'un racisme plus culturel, fondé sur un discours autant que sur un ensemble d'actes, un racisme servant de ciment à une partie de la communauté nationale en France (Balibar / Wallerstein 1997: 28). ${ }^{3}$

La perspective comparée confirme l'importance et la pertinence de la métaphore de 
l'Atlantique noir pour l'analyse d'œuvres, de parcours politiques et de concepts. Envisagée à partir du Sud global, l'émancipation requiert un espace analytique dépassant le cadre national. C'est que ces auteurs, nous le verrons, n'ont eu de cesse d'envisager celle-là tant au niveau local que global. L'Atlantique s'impose dès lors non comme une ressource parmi d'autres, mais comme une nécessité face à la nature de l’objet étudié. Il s'agit, on le sait, d'un espace dynamique, aux contours flous et qui, à ce titre, favorise des processus identificatoires transnationaux. Nous reviendrons sur ce lien entre espace atlantique et identités avant d'examiner la notion d'émancipation. Notre objectif principal vise non à en proposer une nouvelle définition, mais à la questionner du point de vue de ceux qui, à partir de l'expérience de diverses formes de violence physique et symbolique, ont lutté pour l'émancipation collective des peuples soumis à l'esclavage et à la colonisation.

\section{Atlantique noir et identité}

Qu'il s'agisse de la Guinée, des Caraïbes ou de l'Amérique du Nord, la réflexion de Cabral, Césaire et Du Bois sur les formes de l'émancipation naît d'une expérience concrète du racisme, de l'injustice et de l'oppression. L'analyse comparative de ces trois auteurs paraît de ce point de vue aller de soi. Néanmoins, il faut bien saisir que leurs travaux, s'ils partent du local de l'expérience, signifient dans le même temps au niveau global, c'est-à-dire que l'on ne peut les réduire à leur contexte de départ ni en faire les penseurs d'une identité homogène propre à un territoire délimité. Gilroy, influencé par Césaire et Du Bois, refusait, on le sait, les essentialismes, les frontières étanches entre communautés, les conceptions homogènes de la nation. À travers les métaphores de l'Atlantique noir et du navire, c'est bien le lien entre identité et nation qui est envisagé à nouveaux frais : "La spécificité de la culture et de la politique moderne de l'Atlantique noir peut être définie, à un certain niveau, par le désir de transcender à la fois les structures de l'État-nation et les contraintes de l'ethnicité et de la particularité nationale" (Gilroy 2010: 40).

Cependant, même au sein de cet espace dynamique, les discours revendiquant une identité noire homogène ne manquent pas; discours essentialistes, éloignés de la complexité du réel et de la multiplicité des expériences, dans lesquels Gilroy voit encore une 
trace de l'héritage intellectuel occidental: "Cet héritage conditionne en particulier la perpétuation de l'aspiration à une identité supposée authentique, naturelle, stable et 'enracinée'” (idem: 55). Or, quels que soient les exemples choisis (musique, littérature), ceux-ci renvoient tous chez Gilroy à l'existence de l'Atlantique noir et remettent en cause "une hypothétique essence raciale" (idem: 142) qui transcenderait tous les contextes. Il ajoute que les créations culturelles noires circulent, sont reprises, reconstruites, réécrites à partir d'un espace tenant peu compte des frontières des États-nations. "La complexité syncrétique des cultures expressives noires justifie à elle seule de résister à l'idée qu'il y aurait dans ces formes une africanité immuable et pure, dont la puissance d'altérité magique provoquerait chaque fois la perception d'une identité absolue" (idem: 150).

On peut dès lors envisager l'Atlantique noir comme source et support d'une identité alternative, non inscrite dans un lieu borné par une culture et une langue, un lieu de sociabilité marqué par le mouvement, la fluidité, l'hétérogénéité. ${ }^{4}$ Patrick Awondo y voit l'émergence d'un afropolitanisme caractérisé par des formes de citoyenneté globale à partir des mondes africains, afro-américains et caraïbes. Cette sociabilité globale, fondée sur Du Bois, Césaire, Fanon et Gilroy, et s'exprimant à partir de grands centres urbains, dépasse "l'ethnicité et la nationalité pour créer une entité nouvelle" (Awondo 2014: 108).

Ce lieu d'une sociabilité nouvelle, lieu où se donne à voir, à lire et à entendre une multitude de biens symboliques, tend à favoriser des identités excentrées, moins marquées par un nous enraciné et exclusif, plus proches d'un rapport itinérant et inclusif au monde et aux autres. Cette sociabilité-là en évoque une autre, décrite par Léonora Miano qui s'appuie également sur une métaphore, celle de la frontière, pour mettre en évidence la façon dont les écrivains issus de l'Atlantique envisagent leur rapport au monde. À cause d'une histoire marquée par l'esclavage et le colonialisme, ceux-ci n'ont pas eu d'autres choix que d'apprendre à vivre entre plusieurs cultures, ce qui a influencé leur rapport à la langue d'écriture ainsi que leurs choix esthétiques.

La frontière, telle que je la définis et l'habite, est l'endroit où les mondes se touchent, inlassablement. C'est le lieu de l'oscillation constante: d'un espace à l'autre, d'une sensibilité à l'autre, d'une vision du monde à l'autre. C'est là où les langues se mêlent, pas forcément de manière tonitruante, s'imprégnant 
naturellement les unes des autres, pour produire, sur la page blanche, la représentation d'un univers composite, hybride. (Miano 2012: 25)

On retrouvera cet univers esthétique composite dans Les Âmes du Peuple Noir. L'œuvre de Du Bois reflète, nous le verrons, quelque chose de l'espace frontalier: hétérogénéité des genres pratiqués, croisement des langues et des cultures, etc. Ce lieu à partir duquel s'exprime Miano correspond à celui à partir duquel Cabral, Césaire et Du Bois ont écrit, l'Atlantique noir, espace ambivalent, à la fois lieu de la tragédie et lieu de la production artistique et littéraire. Cette identité frontalière n'est d'ailleurs possible que dans certaines régions du monde, Caraïbes, Amériques et Afriques: "La frontière évoque la relation. Elle dit que les peuples se sont rencontrés, quelques fois dans la violence, la haine, le mépris, et qu'en dépit de cela, ils ont enfanté du sens" (ibidem).

Atlantique noir, afropolitanisme, frontière, ces notions désignent à la fois une manière d'être au monde, une pratique particulière de l'art, un espace marqué dans sa genèse par l'exploitation, l'hyperviolence, l'esclavage et le colonialisme. Il s'agit également d'un espace mouvant où trouvent à s'exprimer des auteurs qui, par leur trajectoire et leur pratique respective, ont découplé le lien empreint de sacralité entre langue, identité et nation. Miano ne diffère guère de Cabral, Césaire et Du Bois sur ce point: "J'écris dans l'écho des cultures qui m’habitent: africaine, européenne, américaine, caribéenne. Tout cela vient naturellement se loger dans mon texte" (idem: 29). ${ }^{5}$

Rappelons que la pensée d'un espace symbolique dynamique, aux limites floues, n'est sans doute possible que dans le contexte de la globalisation contemporaine, ainsi que le souligne Gruzinski dans son travail sur les histoires connectées. Ainsi lorsqu'il explore l'espace de la "monarchie catholique" (1580-1640) dans le sillage de Braudel et de Chaunu, l'historien avance l'importance du contexte épistémologique où il travaille:

Le processus de globalisation est en train de modifier inéluctablement les cadres de notre pensée et, par conséquent, nos manières de revisiter le passé. Nous sommes journellement confrontés à des circulations de toutes sortes entre toutes les parties du globe, et donc conduits non seulement à réfléchir sur des questions de "contacts" et de "recouvrements", mais également sur la centralité de notre 'vieux monde' et de ses conceptions. (Gruzinski 2001: 89) 
Cette "monarchie catholique", c'est-à-dire le vaste espace atlantique dominé par le Portugal et l'Espagne lors de la première globalisation, recouvre en partie l'espace auquel nous avons affaire ici. À l'instar de l'Atlantique noir, on y trouve une circulation intense de personnes, de biens symboliques et de marchandises. Et c'est par le biais de cette circulation que des trajectoires, des livres, des concepts acquièrent de nouvelles significations qu'une lecture locale (c'est-à-dire nationale) ne permettait pas de percevoir. Il est donc difficile d'établir des "frontières" dans ce contexte car cela (hommes, idées, marchandises) circule tout le temps et en tous sens.

Elle recouvre un espace qui réunit plusieurs continents, met en rapport ou télescope des formes de gouvernement, d'exploitation économique et d'organisation sociale, confronte parfois très brutalement des traditions religieuses que tout oppose. En ce sens, la monarchie n'est pas une "aire culturelle", elle en rassemble de multiples. (idem: 91-92)

En retravaillant les termes de la première modernité à partir de l'Atlantique espagnol et portugais, Gruzinski pense toutefois qu'il serait erroné de voir dans cette approche une nouvelle preuve de l'occidentalisation du monde. C'est que les savoirs circulent aussi en sens inverse, du Sud vers le Nord, et même selon un axe Sud-Sud. Des textes imprimés à partir de l'Amérique latine, des hommes, des fragments de savoirs autres parviennent toujours plus nombreux en Occident. La compression des distances favorise ainsi une révolution épistémologique: "l'inconnu devient familier, l'inaccessible devient disponible et le lointain relativement proche" (idem: 94). L'une des conséquences de cette circulation-révolution renvoie encore à ce que nous avancions à propos des identités hétérogènes fomentées par l'Atlantique noir. "Partout se mettent en place des systèmes composites de domination et d'organisation du travail, des ensembles de savoirs et de techniques aux origines multiples, des représentations hybrides du soi, de l'espace et du temps, des mélanges de croyances" (idem: 115). C'est au sein de cet espace ambigu, ambivalent, riche de multiples déterminations et significations que nous envisagerons donc la notion d'émancipation. 


\section{Une notion complexe}

L'émancipation comme concept et comme pratique est présente dans le discours critique aussi bien chez des intellectuels du Nord que du Sud. La notion fait retour mais comme le signalent Cukier, Delmotte et Lavergne (2013), elle le fait dans un contexte particulier, celui de la diversité des luttes politiques et de la métamorphose de la critique sociale contemporaine. Comment, dès lors, définir l'émancipation alors qu'elle a gagné des sens divers, presque contradictoires? En outre, la notion a été discréditée, entre autres choses, parce que certains grands projets d'émancipation (e.g. les luttes anticoloniales, le Communisme) ont vu leur part d'utopie trahie dans les faits, la déception débouchant alors sur une remise en cause de la pertinence du concept. Même si, à cause de la diversité des situations où elle trouve à s'appliquer, il peut paraître difficile de donner une définition consensuelle de l'émancipation, Cukier, Delmotte et Lavergne s'en rapprochent en insistant sur l'affranchissement individuel ou collectif par rapport à un lien de tutelle ou d'asservissement: "On associe le plus souvent l'émancipation à une forme de libération, de déprise de rapports de domination, ou bien à l'affirmation et au renforcement d'une puissance d'agir collective ou individuelle, en encore, dans une version légaliste, à la conquête de nouveaux droits" (Cukier et alii 2013: 11). ${ }^{6}$

Quant à la disparité des contextes historiques et sociaux, ils n'envisagent pas d'articuler ceux-ci entre eux par le biais de la traduction linguistique et interculturelle. Certes, disent-ils, il peut y avoir des convergences, des similitudes entre les luttes, mais l'émancipation "n'aura pas la même signification pratique, stratégique et historique particulière" (idem: 12). Les enjeux sont différents ainsi que les pratiques culturelles, ce qui semble être un frein car il est difficile de concevoir que les luttes partout dans le monde convergent "dans un sens unique de l'histoire" (ibidem). Si l'on tente de les rapprocher au nom de l'émancipation, "cela peut donner lieu à des désaccords théoriques, sociaux et politiques" (ibidem). ${ }^{7}$

En cela, ils ne tiennent pas compte d'un facteur dont Cabral, Césaire et Du Bois avaient déjà pointé les effets, à savoir que le capitalisme et le libéralisme, indépendamment des réalités sociales locales, ont produit partout les mêmes effets d'exclusion et de violence 
au détriment des prolétaires, des femmes, des esclaves, des populations colonisées et ce au profit d'une aristocratie bourgeoise, mâle et blanche. Le philosophe Domenico Losurdo a montré que le soi-disant universalisme des valeurs libérales n'a englobé dès l'émergence de cette pensée et représentation du monde que la bourgeoisie européenne. La démocratie libérale ne s'exerce dès lors que pour une caste, au sein d'un espace social strictement délimité. Le libéralisme institué par “la communauté des hommes libres" s'applique à l'intérieur d'un espace sacré qu'une frontière étanche sépare d'un espace profane où les serviteurs/esclaves/peuples colonisés se trouvent mêlés en un tout indistinct.8 "Nous pouvons dire alors que la tradition libérale est la tradition de pensée qui a délimité avec le plus de rigueur un espace sacré restreint où s'appliquent les règles de la limitation du pouvoir" (Losurdo 2013: 345). Quant aux crimes commis tant en Europe (les Irlandais, les prolétaires, les femmes et les enfants pauvres) qu'à l'extérieur des frontières de celle-ci, ils ne sont en rien de regrettables dérives, mais au contraire la conséquence directe d'une représentation raciste et élitiste du monde. "De même qu'outre-Atlantique, la classe dominante est séparée des esclaves noirs et des Noirs en général, en Europe un fossé aux connotations ethniques et raciales sépare la classe dominante des serviteurs blancs" (idem: 109).9

L'intellectuelle et activiste malienne Aminata Traoré pointe aussi la responsabilité du capitalisme et de la représentation libérale du monde dans l'émergence d'une souffrance commune dans le Sud global, mais aussi entre le Nord et le Sud:

Nous sommes nombreux à travers le monde à avoir le sentiment que, par-delà les frontières et les différences de fortune, la réalité du pouvoir est sensiblement la même partout, faite d'indifférence envers les vues et les aspirations profondes des peuples et de fuite en avant dans des solutions qui peuvent s'avérer désastreuses. [...] La concentration du pouvoir entre les mains d'une poignée d'hommes en dit long sur l'impérieuse nécessité de faire naître une citoyenneté transfrontalière; celle qui s'exerce à l'échelle nationale est d'une portée plus que limitée. (Traoré 2002: 168-169)

Face à ce qu'ils tiennent pour un problème insoluble, le lien entre les luttes, Cukier, Delmotte et Lavergne n'envisagent donc pas de recourir au difficile exercice de la 
traduction linguistique et interculturelle. En ce cas précis, l'approche du sociologue Sousa Santos montre que les processus émancipatoires à l'œuvre partout dans le monde non seulement peuvent, mais gagnent à se comprendre et à s'articuler dans leur lutte commune contre le capitalisme, le colonialisme et le patriarcat (Santos 2014: 212). Ajoutons que la traduction est même souhaitable au sein d'une Europe où cohabitent, à côté du récit hégémonique national, les récits des groupes sociaux issus des diasporas; des récits qui, comme le souligne Bentouhami dans le sillage de Stuart Hall, favorisent un rapport narratif autre à la nation. Afin d'assurer la coexistence pacifique entre ces récits et la grande histoire nationale, il faut négocier ce que l'on peut traduire ou pas, estimer le niveau de traductibilité de ces différents récits. ${ }^{10}$ Même si la possibilité de l'échec fait partie de toute opération de traduction, celle-ci a le mérite, à l'inverse de ce que défendent Cukier, Delmotte et Lavergne, de favoriser le rapprochement entre les luttes pour l'émancipation menées dans des contextes spatiaux et temporels distincts.

Because there is no single universal social practice or collective subject to confer meaning and direction to history, the work of translation becomes crucial to identify, in each concrete and historical moment or context, which constellations of practices carry more counterhegemonic potential. (Santos 2014: 222)

Il nous semble que la critique sociale eurocentrée gagnerait à comprendre ce que cette opération de traduction en vient à signifier véritablement en faisant retour à Cabral, Césaire et Du Bois, trois penseurs engagés qui, sans en avoir toujours eu conscience, ont rapproché des pratiques sociales et des luttes pour l'émancipation tout en se jouant des frontières entre les disciplines, ainsi qu'entre science et littérature puisque tous furent à la fois essayistes, poètes (Cabral, Césaire), dramaturge (Césaire) ou romancier (Du Bois).

\section{Envisager l'émancipation à divers niveaux}

Pour Cabral, Césaire et Du Bois, l'émancipation doit être perçue à la fois dans sa dimension collective (la libération des peuples opprimés) et individuelle (la déprise chez l'individu et par l'individu des rapports d'aliénation, qu'il s'agisse des rapports de races, de 
genres ou de classes). Il faut entendre cette double dimension non dans un rapport temporel de succession mais de simultanéité: ainsi chez Du Bois et Cabral, l'émancipation politique et légale du peuple opprimé va-t-elle clairement de pair avec l'émancipation de l'individu par la culture. Le premier défendait l'émancipation des Afro-américains en tant que groupe (et non classe) opprimé, sans toutefois perdre de vue l'importance de l'émancipation individuelle par le biais de l'enseignement: "Au-delà de notre socialisme moderne et à côté du culte pour les masses, doit persister et évoluer cet individualisme plus élevé que protègent ces centres de culture [il parle de l’Université noire]" (Du Bois 2007: 107). ${ }^{11}$

On sait l'influence que Du Bois a exercée sur les penseurs africains et noirs de la diaspora ainsi que sur l'émergence d'une théorie critique atlantique. Son maître ouvrage, Les Âme du Peuple Noir, a en effet eu une portée qui a dépassé les États-Unis depuis longtemps. Publié en 1903, le livre, ensemble hybride fait d'articles, d'essais sociologiques et de textes littéraires, a connu pas moins de cent-dix-neuf rééditions (aux États-Unis) en un siècle, preuve de la nécessité renouvelée de son contenu. Ouvrage hybride dès l'abord, reflet de l'éclectisme intellectuel d'un auteur versé en histoire comme en sociologie, philosophie, ou encore en études classiques, il porte également les marques de l'intérêt de Du Bois pour les arts et l'esthétique dans ses seuils et paratextes puisque chaque chapitre s'ouvre sur un exergue d'un auteur anglais, allemand ou persan ainsi que sur un extrait de Chants de Douleur. À cet égard, l'homologie avec un Atlantique noir aux frontières floues est ici patente. En d'autres mots, l'hétérogénéité esthétique de l'œuvre renvoie à celle de l'espace symbolique où elle s'inscrit.

Il semble que face à la permanence de l'aliénation, de l'oppression, de la domination sociale, économique et culturelle blanche quarante ans après l'abolition de l'esclavage, il fallait à Du Bois dire la réalité des affranchis d'une façon autre, radicalement neuve. En cela, il a influencé bon nombre d'autres penseurs africains et noirs issus des diasporas africaines des $\mathrm{XX}^{\mathrm{e}}$ et XXIe siècles qui, de Césaire à Achille Mbembe en passant par Frantz Fanon et Aminata Traoré, ont toujours accordé une grande importance à la forme de leurs essais, à 
une nouvelle manière de dire le monde qui ne soit pas (trop) marquée par la langue et la culture de l'ancien maître.

Au moment où il écrit son livre, Du Bois prend acte des apories de l'émancipation: émancipés en droits, les affranchis restent encore aliénés dans de nombreux domaines. Les ex-esclaves et leurs descendants continuent, dit-il, à dépendre d'une structure économique, sociale et culturelle laissant peu de marges de manœuvre. Il avait saisi que le statut d'homme libre ne recouvrait pas toute la signification de l'émancipation. Libérer sans redistribuer les terres, libérer sans attribuer le droit de vote et de représentation, libérer sans faciliter l'accès à la propriété ou à l'éducation finit par réduire la portée de l'émancipation première. Comme il l'avance à plusieurs reprises, il a été dommageable pour le Noir d'être passé du statut d'esclave à celui d'homme libre dans un système économique capitaliste où il devenait un concurrent bon marché pour le travailleur blanc pauvre. La lutte pour l'émancipation du peuple noir ne s'achevait donc pas avec l'abolition formelle de l'esclavage. Selon Du Bois, il fallait pour atteindre ce but déconstruire les représentations qui continuaient à dominer, et à structurer en grande partie, les mondes blanc et noir.

Bien sûr, reconnait-il, les gens se côtoient mais "malgré de nombreux contacts physiques et des mélanges quotidiens, il n'y a presque aucune communauté de vie intellectuelle, aucun point de transfert" (idem: 175) entre les pensées et les sentiments des Noirs et des Blancs. L'homme blanc est autant prisonnier de la ligne de partage que le Noir car quand des citoyens blancs éclairés tentent de construire quelque chose en collaboration avec leurs homologues noirs, quelqu'un a toujours "mis en avant, de force, la question de la couleur, et a opposé la puissance énorme de la loi non écrite aux innovateurs" (idem: 176).

De ce point de vue, il avait conscience du rôle joué par les représentations du Noir dans les différents appareils idéologiques du monde blanc. Bien sûr, admet-il, le niveau social et culturel du monde noir empêche celui-ci de jouir pleinement de ses droits civiques, mais le préjugé dont il est l'objet "est plus souvent une cause qu'une conséquence de l'avilissement des Noirs" (idem: 57). Ce préjugé raciste est savamment "entretenu et nourri à tous les niveaux du corps social, depuis l'Associated Press jusqu'à la Church of Christ" (ibidem). C'est que le corps noir émancipé par la justice blanche continue à porter les traces 
de son inscription dans le monde sur le mode des préjugés; autrement dit, il est signifié, couvert de signes, de représentations, qui le déterminent malgré tous les efforts de l'exesclave pour en démontrer l'inanité.12

Frantz Fanon avait aussi compris que l'émancipation signifiait bien autre chose que la simple déprise légale d'un corps soumis à son maître. Pour Elsa Dorlin, dans une relecture de Fanon, l'émancipation comporte “une dimension légaliste, c'est-à-dire qu'être émancipé correspond avant tout à un statut juridique" (Dorlin 2013: 233). On se trouverait alors davantage du côté de l'octroi de droits de la part du pôle dominant au pôle dominé. À l'inverse, "Fanon s'inscrit dans une tradition qui reprend le concept de libération comme explosion de mouvement, y compris de mouvement par et dans la violence, sans attendre d'être autorisé à être libre" (idem: 234). Dans cette place accordée au corps cherchant à se libérer se joue quelque chose d'essentiel car pour l'ex-esclave comme pour l'ex-colonisé, le racisme a déterminé une façon pour son corps d'être au monde.

Je pense donc que toute la question du hors de soi renvoie à ce problème: comment on construit un autre schéma corporel, comment on se schématise pour ainsi dire, comment quelque chose de l'ordre d'un “je incarné” s'expérimente et se redessine, se reconfigure, se délimite dans l'espace et dans le temps et institue une autre historicité-matérialité du sujet. (idem: 238)

C'est bien de cela dont il s'agit chez Cabral, Césaire et Du Bois: réinscrire le corps de l'ex-esclave et de l'ex-colonisé dans un autre rapport à soi et à l'autre, non pas successivement mais simultanément. Dans son Discours sur le Colonialisme, Césaire a ainsi montré combien l'inscription du colonisé dans le monde était en partie déterminée par le colonisateur et sa culture: en Afrique une ligne de couleur se dresse entre les deux pôles et se reproduit par le biais de l'épistémologie, des représentations et des sciences issues du monde occidental. L'ennemi n'est donc pas seulement le banquier, le capitaliste, le militaire, mais tous ceux qui contribuent à la colonisation des esprits, journalistes, écrivains, essayistes (Césaire 2004: 38). Qu'ils soient bien ou mal intentionnés importe peu, "l'essentiel étant que leur très aléatoire bonne foi subjective est sans rapport aucun avec la portée objective et sociale de la mauvaise besogne qu'ils font de chiens de garde du 
colonialisme" (idem: 39). Césaire rappelle de la sorte que le colonialisme est fondé sur des constructions textuelles (géographie, philosophie, religion, sociologie...) partant de présupposés raciaux et faisant peu de cas des critères scientifiques de validité d'une hypothèse. ${ }^{13}$

Et voici la saisissante unité de tout cela, la persévérante tentative bourgeoise de ramener les problèmes les plus humains à des notions confortables et creuses: l'idée du complexe de dépendance chez Mannoni, l'idée ontologique chez le R.P. Tempels, l'idée de "tropicalité” chez Gourou. Que devient la Banque d'Indochine dans tout cela? Et la Banque de Madagascar? Et la chicote? Et l'impôt? (idem: 51)

Tout cela, dit-il, disparaît, rendu " méconnaissable au royaume des pâles ratiocinations" (ibidem). L'écrivain martiniquais insiste sur la violence du système et de ses pratiques mais, ajoute-t-il, sans un vaste système discursif tendant soit à le justifier soit à le nier, le système en question n'aurait pu perdurer.

C'est donc parce qu'ils avaient saisi l'essence de l'esclavage et du colonialisme que Cabral, Césaire et Du Bois défendaient la nécessité d'une émancipation portant sur les deux pôles, la disparition d'une série de contraintes pesant sur les ex-esclaves ainsi que sur les populations colonisées devant en retour libérer les populations blanches et métropolitaines d'une représentation raciste du monde. On le sait, Du Bois envisageait deux voies principales censées favoriser l'émancipation des Noirs américains, le droit de vote et de représentation d'une part et l'accès à l'enseignement universitaire d'autre part. La portée de l'accès à l'université allait bien au-delà de la seule émancipation des populations noires puisque l'ignorance, principe explicatif premier de la permanence du racisme, touchait aussi bien les Blancs du Sud des États-Unis. Sa conception de l'enseignement à l'orée du XXe siècle paraîtra à beaucoup utopique, surtout si l'on veut bien se rappeler les errements de ce siècle-là, mais, à bien des égards, l'horizon utopique de Du Bois pourrait également constituer le nôtre tant le racisme culturel, et dans une moindre mesure le racisme biologique, continue à peser dans le monde occidental: 
Une formation qui permette d'utiliser au mieux le travail humain sans réduire en esclavage ou sans brutaliser; une formation qui nous donne du poids pour encourager les opinions qui servent de rempart à la société, pour éradiquer les préjugés qui, par leur barbarie, font hurler les âmes prisonnières du Voile au point de nous assourdir - pour contenir la fureur montante des hommes enrayés par leurs fers. (Du Bois 2007: 92)

Envisagée de la sorte, la question de l'éducation peut paraître un truisme, il faut toutefois garder à l'esprit combien elle était essentielle dans un contexte social où il fallait apprendre à des hommes blancs et noirs "à vivre ensemble de manière profitable" (ibidem). Dans le contexte spécifique du Sud des États-Unis, la chose était sans doute plus difficile dans la mesure où l'esclavage avait créé d'une part “deux peuples arriérés" et, d'autre part, une population blanche dont la représentation du monde était structurellement déterminée par le racisme. Expériences maladroites et tâtonnements expliquent que, malgré quelques progrès, il y eut en ce domaine beaucoup d'échecs, mais - Du Bois envisage toujours la complexité d'un problème social dans son interaction avec d'autres problèmes connexes -, cela s'explique en partie par un contexte économique où la concurrence entre travailleurs blancs et noirs, surtout parmi les plus pauvres, était restée forte. Développer le droit du peuple noir à accéder à l'université, à dépasser, en partie, par l'enseignement et la culture, le déterminisme social, revenait également à libérer les Blancs de leurs préjugés à l'égard des ex-esclaves.

Césaire pensait également que faire retour au colonialisme, en démonter les structures profondes, contribuerait, d'une part, à la libération des peuples africains sous domination coloniale et, d'autre part, à celle des peuples qui, en métropole, vivaient enfermés dans une représentation coloniale du monde. Mais il y a plus, car revenir pour les Européens sur ce qu'ont été la conquête et l'occupation coloniales signifiait, plus fondamentalement de leur point de vue, saisir l'origine de l'autre génocide, celui commis par des Européens contre d'autres Européens.

"Il faudrait d'abord étudier comment la colonisation travaille à déciviliser le colonisateur, à l'abrutir au sens propre du mot, à le dégrader, à le réveiller aux instincts enfouis, à la convoitise, à la violence, à la haine raciale, au relativisme moral" (Césaire 2004: 
12). Il ajoute que tous les crimes commis dans les colonies débouchent sur ceci: "il y a le poison instillé dans les veines de l'Europe, et le progrès lent, mais sûr, de l'ensauvagement du continent" (ibidem). Césaire pose ici le lien entre colonialisme et nazisme, stade ultime de la barbarie européenne, et oblige de la sorte la bourgeoisie européenne à comprendre les ressorts du génocide premier: “on l'a absous, on a fermé l'œil dessus, on l'a légitimé, parce que, jusque-là, il ne s'était appliqué qu'à des peuples non européens" (idem: 13). En prenant conscience des crimes commis au nom de la civilisation à l'encontre des esclaves et des Africains, les Américains blancs et les Européens non seulement entendraient leur histoire d'une autre façon mais saisiraient les origines de la situation sociale des Afro-américains et des colonisés, ce qui, in fine, les mèneraient à un autre rapport à soi, à une émancipation ontologique relativement à la représentation raciste du monde.

Cela Cabral l'avait aussi perçu, lui qui a toujours soutenu que la lutte de l'opprimé contre l'oppresseur aurait des conséquences dans les métropoles européennes puisque celles-ci découvriraient que par la résistance culturelle, politique et armée, l'opprimé existe et qu'il désire, par-dessus tout, malgré l'oppression, son émancipation. La nature de la réaction et son intensité dépendent, nous dit Cabral, du "grau de cristalização de uma mentalidade colonialista ou racista das diferentes categorias sociais, isto é, dos indivíduos" (Cabral 2008: 233). Découvrir que le peuple colonisé lutte, qu'il possède une culture propre ainsi qu'un projet nationaliste a donc également un impact sur la population métropolitaine qui a alors l'opportunité de s'émanciper de "um elemento negativo da sua cultura: o preconceito da supremacia da nação colonizadora sobre a nação colonizada" (idem: 234).

On notera encore que si les trois penseurs recourent souvent à la culture noire et africaine comme outil et support de l'émancipation pour les ex-esclaves et leurs descendants ainsi que pour les colonisés, il ne s'agit pas d'une conception de la culture comme figée, centrée sur elle-même, émancipatrice par nature. Si Cabral, Césaire et Du Bois défendent un retour à certaines pratiques sociales et expériences historiques africaines produites comme non-existantes ou dévalorisées par le savoir-pouvoir aux États-Unis et en Europe, il ne s'agit en aucun cas du retour nostalgique à une réalité idéale. Ainsi lorsque Césaire affirme à propos des sociétés africaines précoloniales que “c'étaient des sociétés pas 
seulement anté-capitalistes, comme on l'a dit, mais aussi anti-capitalistes. C'étaient des sociétés démocratiques, toujours" (Césaire 2004: 25), il ne faut pas y voir les traces d'un retour nostalgique. ${ }^{14}$ Les abus existaient de la part de certaines élites qui se sont d'ailleurs entendues avec le colonisateur pour perpétuer des rapports de sujétion et se maintenir au pouvoir: "il s'est établi, au détriment des peuples, un circuit de bons services et de complicité." (idem: 24). De son côté, Cabral soutenait que le retour aux sources africaines, à des expériences sociales ignorées, ne pouvait s'opérer que de façon critique à cause de la diversité des sources en question et de l'hétérogénéité des situations où ce retour aurait lieu: "É um processo lento, descontínuo e desigual, cujo desenvolvimento, ao nível de cada indivíduo, depende do grau de aculturação, das condições materiais de existência, da formação ideológica e da própria história enquanto ser social" (Cabral 2008: 217).

Ce retour leur semblait nécessaire afin de donner à comprendre que les groupes sociaux colonisés étaient porteurs d'une histoire plus riche que ce que supposaient les colonisateurs. Toutefois, parce qu'il a lieu dans le contexte de la lutte pour la libération nationale et que cette lutte produit elle-même de la culture, de nouvelles manières d'être au monde, le retour sera critique (ainsi pas question pour Cabral de reprendre le modèle gérontocratique de certaines sociétés précoloniales ou encore d'accepter un rôle naturellement subalterne pour la femme). En conséquence, revenir à la culture signifie revenir dialectiquement à celle-ci et entendre que la culture peut être réactionnaire et révolutionnaire, traditionnelle et transformatrice, stagnante et dynamique (Rabaka 2010: 265). $\cdot 15$

Ce retour à certaines sources ainsi qu'à une histoire de l'oppression du point-de-vue des opprimés ouvrait pour ces penseurs de nouvelles perspectives critiques, une manière autre de travailler à l'émancipation des damnés de la terre, ce qui impliquait une prise de distance relativement aux pensées eurocentrées de l'émancipation. Ainsi Du Bois devait-il mettre en évidence les apories du marxisme et du socialisme sur le point précis de l'émancipation du travailleur de couleur. On retiendra qu'il a fait partie pendant un an du parti socialiste américain (1911-1912), mais qu'il a quitté celui-ci à cause du racisme présent au sein de ses structures (exclusion des membres noirs et asiatiques). Cela 
confirme ce qu'il avait mis en évidence dans un texte de 1907 (The Negro and Socialism) portant sur les contradictions du socialisme, contradictions que Rabaka a synthétisées de la sorte:

a silence on and/or inattention to: race, racism, and anti-racist struggle; colonialism and anti-colonial struggle; and, the ways in which both capitalism and colonialism exacerbate not simply the economic exploitation of non-European peoples, but continues (both physical and psychological) colonization beyond the realm of political economy. (Rabaka 2010: 52)

Selon Du Bois, le socialisme s'attache avant tout à défendre les droits du travailleur blanc, de sexe masculin, ce qui, soulignons-le au passage, rappelle combien l'écrivain et intellectuel était conscient des liens étroits entre capitalisme, racisme et relation inégale de pouvoir entre les sexes. Située à l'intersection de diverses formes d'oppression, la femme américaine noire aurait pu, à cause de sa situation, revendiquer une place centrale dans le processus d'émancipation, notamment au sein des organisations féministes nordaméricaines. Cependant, Du Bois avait été frappé de voir le racisme à l'œuvre dans les associations féministes (notamment à la National American Woman Suffrage Association) réclamant le droit de vote pour les seules femmes blanches. Or, dès 1898 (The Study of Negro Problems), il avait pourtant mis en évidence que l'émancipation des un(e)s favoriserait inévitablement l'émancipation des autres: "Every argument for Negro suffrage is an argument for woman's suffrage; every argument for woman's suffrage is an argument for Negro suffrage; both are great movements in democracy" (Du Bois apud Rabaka 2010: 64). Il faut donc rappeler une dernière fois que pour Du Bois, il allait de soi que l'acquisition d'un droit civique ou social pour les Noirs américains amplifierait la portée de l'émancipation à d'autres segments sociaux, les prolétaires blancs et les femmes blanches notamment. On voit ainsi se dessiner un projet d'émancipation qui est aussi un projet de démocratie la plus inclusive possible. À l'intersection de notions comme la classe, le genre et la race, $\mathrm{Du}$ Bois a pensé une démocratie en expansion permanente, transgenre et interethnique. 
L’analyse que fera Cabral des rapports déséquilibrés de pouvoir entre races, classes et sexes ne diffère guère de celle de Du Bois et de Césaire. Il a en effet toujours articulé le local (la lutte contre le colonisateur portugais en Guinée) avec le global (la lutte contre toute forme d'oppression), la critique du capitalisme avec celle du racisme d'origine coloniale. Selon lui, une théorie critique du capitalisme devait dépasser la critique du capitalisme dans le monde occidental, les théories globales issues du monde occidental finissant par ne tenir compte que des problèmes propres aux sociétés industrielles et techno-capitalistes, alors que Cabral englobe le monde colonial dans sa critique et offre de la sorte une vision plus subtile et plus pertinente des relations inégales de pouvoir au niveau global. ${ }^{16}$ Avec Cabral, comme avec Césaire d'ailleurs, la lutte pour l'émancipation s'inscrit dans le local de la lutte concrète (armée dans le cas de la Guinée Bissau) et est comprise comme une lutte globale, visant à l'émancipation de tous. En d'autres mots, il ne s'agit pas uniquement de lutter contre le colonialisme portugais pour l'émancipation des Guinéens et des Cap-Verdiens. Il part d'une expérience nationale concrète pour défendre un nationalisme d'ouverture, un nationalisme humaniste, un humanisme révolutionnaire. La lutte pour l'émancipation ne devait pas non plus s'arrêter avec l'indépendance du Cap-Vert et de la Guinée: elle devait se poursuivre au niveau du continent ainsi que des autres peuples sous domination impérialiste (notamment les Palestiniens). ${ }^{17}$

La question que l'on est en droit de se poser face à cet élargissement permanent de l'émancipation à d'autres cercles est celle de ses limites: jusqu'où faire porter celle-ci? À suivre Cabral, Césaire et Du Bois, il faut en élargir sans cesse la portée. Si elle a commencé en faveur des droits des populations noires, l'émancipation, défend Césaire, touche à "l'humanité tout entière". En d'autres termes, on ne peut s'arrêter dans la lutte, il faut sans cesse l'articuler à d'autres: “Notre engagement n'a de sens que s'il s'agit d'un réenracinement certes, mais aussi d'un épanouissement, d’un dépassement et de la conquête d'une nouvelle et plus large fraternité" (Césaire 2004: 92). ${ }^{18}$ Reprendre Cabral, Césaire et Du Bois à partir de l'Atlantique noir signifie, comme nous le soulignions d'entrée de jeu, rapprocher les luttes, utiliser le biais de la traduction interculturelle afin de comprendre ce qui les unit et ce contre quoi elles s'organisent. Le rapprochement entre savoirs et pratiques 
favorisant l'émancipation sociale repose en grande partie sur un travail, aussi indispensable que fragile, de traduction, qui, entre autres apports, met en évidence les apories, contradictions et impasses de la modernité occidentale (Santos 2014: 233).

Ce travail n'est possible, comme nous l'avons vu, qu'à partir du point de vue de ceux qui ont douloureusement et concrètement fait l'expérience des contradictions en question. Après Cabral, Césaire et Du Bois, d'autres intellectuels issus des mondes atlantiques, ont poursuivi la lutte pour l'émancipation, notamment en traduisant pour le bien commun le projet porté par le capitalisme, à partir justement de cet autre lieu. Ainsi Aminata Traoré, qui revendique pleinement l'héritage de Césaire, a-t-elle proposé de "réécrire, relire et redire le projet néolibéral en Afrique" à travers un exercice de traduction au terme duquel la représentation néolibérale du monde se trouve complètement remise à plat. Vues à partir du Sud, voici ce que deviennent certaines notions centrales pour la représentation libérale du monde:

Libre-échange: Liberté de circulation des capitaux, des biens et des services au profit des principales puissances économiques et d'une minorité d'Africains, mais refus de la libre circulation des êtres humains. Croissance: création de richesse au détriment des pauvres et de l'environnement. Terrorisme: terme utilisé pour désigner la violence perpétrée sans l'aval des puissants de ce monde et contre leurs intérêts. (Traoré 2002: 183-185)

Traduire signifie de la part de l'intellectuelle malienne un exercice herméneutique (comprendre la vision capitaliste et libérale du monde) et un engagement pratique (soutenir une émancipation alternative à partir d'expériences et de savoirs dévalorisés par la raison occidentale), dont nous avons trouvé les traces premières dans le travail de Cabral, Césaire et $\mathrm{Du}$ Bois. Le rapprochement entre ceux-ci montre encore que les œuvres produites dans l'Atlantique noir demandent presque naturellement une perspective comparée. Il ne s'agit bien sûr pas de dénier la possibilité d'une réception/lecture locale, mais de rappeler que le sens de ces œuvres ne s'épuise pas dans celle-ci. De ce point de vue, les réflexions de Cabral, Césaire et $\mathrm{Du}$ Bois relatives à la nature et à la portée de l'émancipation aident sans doute à mieux saisir les significations de biens symboliques 
marqués par l'hyperviolence, la coercition et l'exploitation. La comparaison atlantique trouve ici toute sa justification. Il revient à l'historien, comme au critique ou au philosophe, d'œuvrer à une autre échelle afin d'opérer ce que Gruzinski appelle des branchements (au sens très concret de l'électricien travaillant sur un réseau) entre zones géographiques, parcours biographiques, livres, etc. Les connexions en question, souvent oblitérées ou altérées pas les histoires nationales, rendent en effet mieux compte de ce qui se joue au sein d'espaces dynamiques tels que l'Atlantique noir ou la "monarchie catholique".

\section{NOTES}

\footnotetext{
${ }^{1}$ Nous avons publié une longue recension critique de ce numéro (Schurmans 2016a).

${ }^{2}$ L'itinérance est une notion essentielle (et ambigüe) pour les approches postcoloniales des littératures du Sud global, que nous avons définie dans le Dictionnaire Alice/Dicionário Alice (Schurmans 2019a).

${ }^{3}$ C'est ce que confirmait en 2013 le journaliste antillais Harry Roselmack, premier présentateur noir du journal télévisé en France, à l'occasion d'un commentaire raciste émanant d'une élue FN. Ce commentaire réduisait la personne visée (Christiane Taubira) à une condition de race, mais pour Roselmack, il allait bien au-delà et évoquait la permanence en France d'une personnalité raciste: “Ce qui me chagrine, c'est le fond de racisme qui résiste au temps et aux mots d'ordre, pas seulement au sein du FN, mais au plus profond de la société française. C'est un héritage des temps anciens, une justification pour une domination suprême et criminelle: l'esclavage et la colonisation" (Roselmack 2013: s.p.). Les commentaires désobligeants à l'encontre de la Garde des sceaux ont été l'occasion pour la presse de revenir sur la libération de la parole raciste dans le champ politique français et européen depuis septembre 2001 (Abdul / De Montvalon 2013: 6; Joie 2013: 12-13). L'une des grandes spécialistes de l'histoire de l'Afrique subsaharienne s'en affligeait dans sa contribution au Livre Noir du Colonialisme:
}

Ces préjugés eurocentrés qui présentent des Noirs ou des Jaunes une image dépréciée demeurent partout en filigrane. [...] Le savoir tout récent de quelques africanistes de bonne volonté est encore impuissant à contrer cet énorme 
héritage que l'on peut qualifier aujourd'hui d'abominable, car il n'a plus pour le justifier ou du moins l'expliquer et le comprendre le contexte économique et scientifique dépassé du siècle colonial. (Coquery-Vidrovitch 2006: 915-917)

${ }^{4}$ Cette conception de l'identité n'est évidemment pas propre à l'Atlantique noir. On veillera cependant à ne pas idéaliser un processus identitaire dynamique, surtout lorsqu'il n'est pas choisi mais subi. À l'ère de la modernité liquide (la globalisation), Zygmunt Bauman montre combien il est difficile pour les marginaux, les relégués, les réfugiés (qu'il regroupe sous la catégorie d'outcasts) de s'insérer dans un espace occidental marqué par un lien fort entre identité individuelle, identité nationale et État. L'identité et le sentiment d'appartenance ne sont plus "secured by a lifelong guarantee", mais négociables, voire révocables pour un nombre croissant de sujets (Bauman 2004: 11).

${ }^{5}$ Les études littéraires postcoloniales ont souvent insisté sur ce découplage langue/nation ainsi que sur les conséquences esthétiques de celui-ci (Moura 2014: 84). À l'instar de Robert Young questionnant les diverses appellations servant à désigner les littératures écrites en anglais à partir du Sud global (2014), Moura constate l'émergence de termes nouveaux, ouvrant certainement d'autres horizons (Littérature-monde, Études de la globalisation), mais sans remettre en cause la spécificité de l'approche postcoloniale des "Littératures du Sud" car elle autorise "une topique fort utile en questionnant les effets coloniaux/impériaux sur les dispositions scripturales et lectoriales, sur les usages des codes littéraires et langagiers, et enfin sur les modes de représentation du réel" (idem: 91). En outre, on retiendra que les approches postcoloniales de la littérature s'ouvrent presque naturellement sur la perspective comparée, cherchant, au-delà de la différence linguistique, à construire de nouveaux espaces où œuvres et parcours d'écrivains en viennent à signifier d'une façon autre (nous avons questionné la catégorie "Littérature africaine lusophone" à partir de ce présupposé, Schurmans 2016b). Les contributions de Young et Moura sont tirées d'un ouvrage collectif (Joubert 2014), que nous avons analysé (Schurmans 2016c).

${ }^{6}$ Le numéro de Manière de Voir consacré à "L'Émancipation dans l'Histoire" montre que celle-ci ne fait sens qu'envisagée au niveau global. Il s'agit souvent d'une utopie, d'un horizon vers lequel tend la lutte, mais d'une utopie incontournable:

Peu de mots résument aussi bien des siècles de combats qu'émancipation. Si, au propre, il s'applique aux mineurs déclarés majeurs, au figuré, il recouvre toutes les formes d'affranchissement - esclaves, masses exploitées, femmes dominées, minorités opprimées, peuples colonisés... Libérer l'être humain de ce qui l'empêche de s'épanouir, voilà ce dont ont rêvé des millions d'hommes et de femmes au fil de l'histoire. (Vidal 2009: 4)

${ }^{7}$ Cette question essentielle de la difficulté éprouvée à articuler entre elles les luttes pour l'émancipation a été posée par Frédéric Lordon lorsqu'il analyse les causes de la crise économique de 2008. Si la solidarité avec d'autres gauches en Europe est souhaitable, il faut, défend-il, d'abord privilégier le national au nom de la communauté de langue. L'expérience montre que dans certains cas concrets, il y a parmi les acteurs engagés dans la lutte "impossibilité totale de se parler, de se comprendre, donc de se coordonner et de lutter". Même s'il ne l'évoque pas, la traduction semble malgré tout inévitable: "Il ne se formera donc pas une gauche - qui serait d'emblée 
postnationale. Il se formera des gauches, localement ancrées et cependant hautement désireuses de se parler et de s'épauler" (Lordon 2014: 19).

${ }^{8}$ Définir la notion de liberté à partir de la tradition philosophique européenne comporte toujours le risque de ne tenir compte que du point de vue exprimé à partir de l'espace sacré dont parle Losurdo. C'est ce que nous avons rappelé dans le Dictionnaire Alice/Dicionário Alice (Schurmans 2019b).

${ }^{9}$ L'historien et économiste libanais Georges Corm souligne la même contradiction dans un ouvrage portant sur la construction de l'Occident comme mythe. Il relève d'un côté "l'appel à la pleine liberté de l'individu" contre une série de dogmatismes et, de l'autre, l'institutionnalisation d'un système économique produisant de l'inégalité au sein et en dehors de l'Europe. C'est le capitalisme libéral "qui encourage le pillage colonial, prive les peuples colonisés de leurs ressources naturelles, transforme leur économie et leur équilibre écologique au profit de la métropole" (Corm 2012: 131).

10 "La traduction est nécessaire en raison de la 'prolifération subalterne de la différence' dont la logique souligne le paradoxe de la mondialisation contemporaine" (Bentouhami 2013: 278), c'est-à-dire d'une part la mondialisation d'un modèle culturel (américain) et la prolifération de particularismes. Il faut alors négocier entre les diverses formes culturelles et entre celles-ci et la culture dominante l'accès au récit national. Cette négociation ressemble à "un exercice de type herméneutique qui vise aussi bien à entendre qu'à se faire comprendre en empruntant ses modes d'expression à la culture d'appartenance" (ibidem). La négociation renvoie encore à la "traductibilité réciproque des cultures", inévitable dans le contexte d'une société marquée par la présence de communautés issues d'ex-colonies.

${ }^{11}$ On retrouve chez Cabral cette même recherche de l'émancipation au niveau collectif et individuel. Sa conception de l'identité explique sans doute en partie pourquoi même s'il tient compte de la notion de lutte des classes, celle-ci ne peut rendre totalement compte de ce qui se joue dans les sociétés colonisées. Pour lui, deux identités se côtoient en tout individu, une identité biologique (ou identité première) et une identité sociologique (ou identité actuelle), entretenant une relation dialectique où le stable le dispute à l'instable, au mouvement: "Com efeito, a identidade não é uma qualidade imutável, precisamente porque os dados biológicos e sociológicos que a definem estão em permanente evolução" (Cabral 2008: 219). Cette relation dialectique, on la retrouvera également à un autre niveau, l'identité selon Cabral étant en effet ce qui identifie un individu ou un groupe et le distingue d'un autre individu ou groupe. L'humanisme de cette conception de l'identité tient à ceci de fondamental: s'il existe des éléments permettant de distinguer les hommes entre eux, il existe également des éléments communs. "A definição de uma identidade, individual ou colectiva, é portanto, simultaneamente, a afirmação e a negação de um determinado número de características que definem indivíduos ou colectividades em função de coordenadas históricas (biológicas e sociológicas), em dado momento da sua evolução" (ibidem).

${ }^{12}$ Cette persistance du signe, de la cicatrice est patente dans certains romans portant sur la traite et l'esclavage issus de l'Atlantique noir. Nous en avons étudié les caractéristiques sous le signe de la postmémoire (Schurmans 2017).

N. 40 - 06/ 2019 | 91-117 - ISSN 2183-2242 | http:/dx.doi.org/10.21747/21832242/litcomp40a4 
${ }^{13}$ Depuis Mudimbe, nous savons que ces travaux en disent davantage sur la façon dont l'Europe coloniale a perçu l'Afrique noire que sur l'Afrique noire elle-même. Les discours anthropologique, philosophique, géographique, etc. relèvent du même savoir-pouvoir: "They speak about neither Africa nor Africans, but rather justify the process of inventing and conquering a continent and naming its 'primitiveness' or 'disorder', as well as the subsequent means of its exploitation and methods for its 'regeneration'” (Mudimbe 1988: 20).

14 “Thus, Césaire's return to Africa is more spiritual and cultural than physical, and it requires a critical (dare I say, dialectical) exploration of the past, which for many continental and, especially, diasporan African means salvaging what we can in the aftermath of the horrors of the African holocaust, enslavement, colonization, segregation, and Eurocentric assimilation" (Rabaka 2010: 130).

${ }^{15}$ Le processus de décolonisation et celui de ré-africanisation, processus révolutionnaires, pose une question essentielle: “what it means - ontologically, existentially, and phenomenologically speaking - to be 'African' - that is, 'African' in a world dominated by European imperialism or, to put it another way, it calls into question what it means to be 'black' in a white supremacist colonial capitalist world" (idem: 265).

16 "As the world actually exists, it is an imperial world, a world where one human group doggedly attempts to dominate all other human groups; where one human culture and civilization is acknowledged and exalted as the only authentic human culture and civilization; where one's people history is considered the 'History' of humanity in toto" (idem: 235).

${ }^{17}$ Pour l'internationalisme, s'émanciper hic et nunc ne se concevait que si l'on travaillait à l'émancipation de l'autre, proche ou lointain. Ainsi ce que montrent bien Faligot (2013) et El Tahri (2007), c'est que la lutte pour l'émancipation menée à partir de Cuba a été une lutte locale et globale, cubaine et atlantique, Castro et Guevara ayant toujours défendu un élargissement des luttes visant à l'émancipation. Les résultats sont connus, soutien massif au renforcement des indépendances algérienne et angolaise et rôle de premier plan dans l'accession de la Namibie à l'indépendance; les échecs également: interventions au Congo et en Bolivie.

${ }^{18}$ Cette position relativement à la portée de l'émancipation semble être une caractéristique inhérente aux luttes menées à partir du continent africain. Achille Mbembe interprète de la sorte le parcours de Nelson Mandela.

Tout comme les mouvements ouvriers du XIX $\mathrm{X}^{\mathrm{e}}$ siècle, ou encore les luttes des femmes, notre modernité aura été travaillée par le rêve d'abolition qu'auront porté auparavant les esclaves. C'est ce rêve que prolongeront, au début du $\mathrm{XX}^{\mathrm{e}}$ siècle, les combats pour la décolonisation. La praxis politique de Mandela s'inscrit dans cette histoire spécifique des grandes luttes africaines pour l'émancipation humaine. (Mbembe 2013: 14)

Ces luttes, rappelle Mbembe, bien qu'ancrées dans le local, ont toujours eu une portée globale puisqu'elles "ont permis l'extension ou encore l'universalisation de droits qui, jusque-là, étaient restés l'apanage d'une race” (ibidem).

N. 40 - 06/ 2019 | 91-117 - ISSN 2183-2242 | http:/dx.doi.org/10.21747/21832242/litcomp40a4 


\section{Bibliographie}

Abdul, Shahazad/ Jean-Baptiste De Montvalon (2013), «La Radicalisation du Discours Politique Légitime le Racisme», Le Monde, vendredi 8 novembre, 7.

Awondo, Patrick (2014), «L’Afropolitanisme en Débat», Politique Africaine, no 136, 105-119.

Balibar, Étienne/ Immanuel Wallerstein (1997), Race, Nation, Classe. Les Identités Ambigües, Paris, La Découverte.

Bauman, Zygmunt (2004), Identity, Cambridge \& Malden, Polity Press.

Bentouhami, Hourya (2013), «Identités et Cultures. Pour un Multiculturalisme Négocié», in Émancipation, les Métamorphoses de la Critique Sociale, Bellecombe-en-Bauges, Éditions du Croquant, 251-284.

Cabral, Amílcar (2008), «0 Papel da Cultura na Luta pela Independência», in Documentário, Lisboa, Biblioteca Editores Independentes, 203-236.

Césaire, Aimé, (2004), Discours sur le Colonialisme, Paris, Présence Africaine.

Coquery-Vidrovitch, Catherine (2006), «Le Postulat de la Supériorité Blanche et de l'Infériorité Noire», in Le Livre Noir du Colonialisme. XVIe-XXIe Siècle: De l'Extermination à la Repentance, Paris, Hachette Littératures, 863-925.

Corm, Georges (2012), L'Europe et le Mythe de l'Occident. La Construction d'une Histoire, Paris, La Découverte.

Cukier, Alexis/ Fabien Delmotte/ Cécile Lavergne (éds.) (2013), Émancipation, les Métamorphoses de la Critique Sociale, Bellecombe-en-Bauges, Éditions du Croquant.

Dorlin, Elsa (2013), «La Violence comme Praxis Libératrice: de Frantz Fanon à l'Autodéfense», in Émancipation, les Métamorphoses de la Critique Sociale, Bellecombe-enBauges, Éditions du Croquant, 231-250. 
Du Bois, W.E.B (2007), Les Âmes du Peuple Noir, éd. Magali Bessone, Paris, La Découverte.

El Tahri, Jihan (2007), Cuba, une Odyssée Africaine. France, 190 min.

Faligot, Roger (2013), Tricontinentale, Paris, La Découverte.

Fouquet, Thomas (org.) (2014), Politique Africaine, «Blackness», no 136.

Gruzinski, Serge (2001), «Les Mondes Mêlés de la Monarchie Catholique et autres ‘Connected Histories'», Annales. Histoire, Sciences sociales, no 1, 56e année, 85-117.

Joie, Catherine (2013), «Les Européens ont Débridé leur Parole», Le Soir, vendredi 29 novembre, 12-13.

Lordon, Frédéric (2014), «La Gauche ne peut pas Mourir», Le Monde Diplomatique, septembre, 1, 18-19.

Losurdo, Domenico (2013), Contre-Histoire du Libéralisme, trad. Bernard Chamayou, Paris, La Découverte.

Mbembe, Achille (2013), «Nelson Mandela, les Chemins Inattendus», Le Monde Diplomatique, août, 14-15.

Miano, Leonora (2012), Habiter la Frontière, Paris, L'Arche.

Moura, Jean-Marc (2014), «Critique Francophone du Postcolonial et Critiques Postcoloniales de la Francophonie», in Le Postcolonial Comparé, Anglophonie, Francophonie, Saint-Denis, Presses Universitaires de Vincennes, 81-95.

Mudimbe, V.Y. (1988), The Invention of Africa. Gnosis, Philosophy and the Order of Knowledge, Bloomington, Indianapolis, London, Indiana University Press and James Currey. Rabaka, Reiland (2010), Africana Critical Theory. Reconstructing the Black Radical Tradition from W.E.B. Du Bois and C.L.R. James to Frantz Fanon and Amílcar Cabral, Lanham, Lexington Books.

Roselmack, Harry (2013), «La France Raciste est de Retour», Le Monde, 
<http://www.lemonde.fr/idees/article/2013/11/04/harry-roselmack-la-france-racisteest-de-retour_3508055_3232.html> (consulté le 05/11/2013).

Santos, Boaventura de Sousa (2014), Epistemologies of the South. Justice against Epistemicide, Boulder, London, Paradigm Publishers.

Schurmans, Fabrice (2019a), «Itinerâncias (Itinérance)», in Dicionário Alice/Dictionnaire Alice, Coimbra, CES \& Alice,

<http://alice.ces.uc.pt/dictionary/?id=23838\&pag=23918\&id_lingua=1\&entry=24308> (consulté le 20/04/2019).

-- (2019b), «Liberdade (Liberté)», Dicionário Alice/Dictionnaire Alice, Coimbra, CES \& Alice. <http://alice.ces.uc.pt/dictionary/?id=23838\&pag=23918\&id_lingua=1\&entry=24314> (consulté le 30/04/2019).

-- (2017), «Vers une Postmémoire de la Traite et de l’Esclavage?», Carnets. Pratiques de l'Errance et Vécus de la Mémoire, no 10, 1-14. http://carnets.revues.org/2188 DOI: 10.4000/carnets.2188 (consulté le 01/02/2019)

-- (2016a), «Politique Africaine, Blackness, no 136, décembre 2014», e-cadernos ces, As Literaturas Africanas de Língua Portuguesa em Perspetiva Comparada, no 26, 167-171, <http://eces.revues.org/2172> (consulté le 02/02/2019).

-- (2016b), «Ler na Fronteira», e-cadernos ces, As Literaturas Africanas de Língua Portuguesa em perspetiva comparada, $\mathrm{n}$-26, 5-29, <http://eces.revues.org/2100> (consulté le 02/02/2019).

-- (2016c), «Joubert, Claire (éd.) (2014), Le Postcolonial Comparé, Anglophonie, Francophonie, Saint-Denis, Presses Universitaires de Vincennes, coll. Littérature Hors Frontière», @nalyses, Revue de Critique et de Théorie Littéraire, vol. 11, no 2, 462-468, <https://uottawa.scholarsportal.info/ottawa/index.php/revue-analyses/article/view/ 1580> (consulté le 02/02/2019). 
Traoré, Aminata (2002), Le Viol de l'Imaginaire, Paris, Fayard \& Actes Sud.

Vidal, Dominique (2009), «La Médaille, son Revers et son Endroit», Manière de Voir, «L'Émancipation dans l'Histoire», no 106, 4.

Young, Robert (2014) «Littérature Anglaise ou Littératures en Langue Anglaise», in Le Postcolonial Comparé, Anglophonie, Francophonie, Saint-Denis, Presses Universitaires de Vincennes, 45-59.

Fabrice Schurmans est titulaire d'un Doctorat en Études Postcoloniales, est chercheur au Centre d'Études Sociales (U. Coimbra). Ses recherches portent sur les littératures postcoloniales du Sud dans une perspective comparée et interdisciplinaire, les littératures francophones du Nord, les questions théoriques postcoloniales (e.g. traduction, frontière, identité). Il a publié deux livres - Michel de Ghelderode. Un Tragique de l'Identité (2011) et $O$ Trágico do Estado Pós-colonial. Pius Ngandu Nkashama, Sony Labou Tansi, Pepetela (2014). La plupart de ses articles sont disponibles sur Academia et Researchgate. Il a également traduit des dramaturges portugais du XXe siècle et publié des nouvelles dans les revues Balises, Bloganozart, Brèves, Harfang, Legs et Littératures, L'encrier renversé, Mot Dit, Muse/Medusa, Nouvelle Donne, Le récit-page, Rue Saint Ambroise ainsi que dans les recueils collectifs Le Train (Le Soupirail 2018), Spectre (Le Grimoire du Faune 2018), Sortilège (Le Grimoire du Faune 2019), Lignes de Fuite (Bancal 2019, sous presse). 